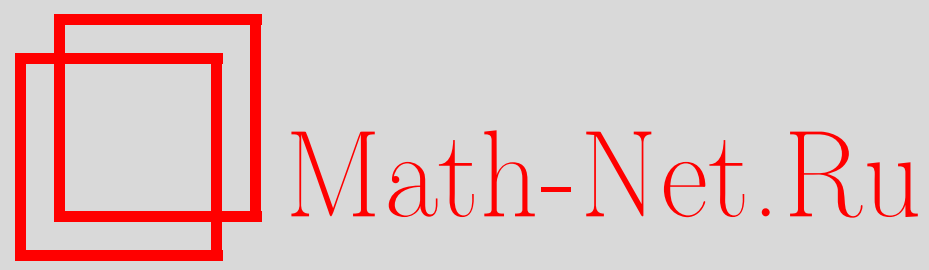

А. Ю. Попов, О проблеме моментов для быстроубывающих функций, Матем. заметки, 1996, том 60, выпуск 1, 66-74

DOI: https://doi.org/10.4213/mzm1804

Использование Общероссийского математического портала MathNet.Ru подразумевает, что вы прочитали и согласны с пользовательским соглашением

http://www . mathnet.ru/rus/agreement

Параметры загрузки:

IP : 54.224 .187 .69

26 апреля 2023 г., 06:38:39




том 60 выпуск 1 июль 1996

\section{О ПРОБЛЕМЕ МОМЕНТОВ \\ ДЛЯ БЫСТРОУБЫВАЮЩИХ ФУНКЦИЙ}

\section{А. Ю. Попов}

Публикация посвящена описанию классов функций $\{f\}$, определенных на $(0,+\infty)$, в которых проблема моментов вида

$$
\int_{0}^{+\infty} t^{\alpha_{n}} f(t) d t=c_{n}
$$

имеет решения при любых правых частях $c_{n}$. Другими словами, произвольная последовательность комплексных чисел $\left\{c_{n}\right\}$ должна быть “допустимой” для решения интерполяционной задачи (1) в одном из упомянутых выше классов.

В [1] Д. Пойа для любой последовательности комплексных чисел $\left\{c_{n}\right\}_{n=0}^{\infty}$ доказал сушествование целой функции $f$ такой, что

$$
\int_{-\infty}^{+\infty} t^{n} f(t) d t=c_{n}, \quad n=0,1,2, \ldots
$$

В [2] был получен следуюший результат. Пусть $S_{+}-$множество всех комплекснозначных функций $\varphi \in C^{\infty}[0,+\infty)$, для которых выполняются соотношения

$$
\begin{gathered}
\forall k \in \mathbb{N}_{0} \quad \varphi^{(k)}(0)=0, \\
\forall k, m \in \mathbb{N}_{0} \quad \lim _{t \rightarrow+\infty} t^{m} \varphi^{(k)}(t)=0 .
\end{gathered}
$$

Тогда любая последовательность комплексных чисел $\left\{c_{n}\right\}_{n=0}^{\infty}$ является моментами на $(0,+\infty)$ некоторой функции $f \in S_{+}$, т.е. выполняются равенства

$$
\int_{0}^{+\infty} t^{n} f(t) d t=c_{n}, \quad n=0,1,2, \ldots
$$

В этой работе доказаны более сильные утверждения. Через $\mathbb{C}^{\infty}$ обозначим множество всевозможных последовательностей комплексных чисел, а через $S_{0}$ - множество всех непрерывных и ограниченных на $(0,+\infty)$ комплекснозначных функций, для которых выполняются соотношения

$$
\forall m \in \mathbb{N} \quad \lim _{t \rightarrow+\infty} t^{m} \varphi(t)=0 .
$$


В частности, для $\forall \varphi \in S_{0}$ и $\forall a \geqslant 0$ сходятся интегралы

$$
\int_{0}^{+\infty} t^{a}|\varphi(t)| d t
$$

Для $\varphi \in S_{0}$ и $q>1$ определим множество функций

$$
\begin{aligned}
E_{\varphi, q}=\left\{f(t)=\sum_{k=0}^{\infty} b_{k} \varphi\left(t q^{-k}\right) \mid\left\{b_{k}\right\}_{k=0}^{\infty}\right. & \in \mathbb{C}^{\infty}, \\
b_{0} & \left.=1, \lim _{k \rightarrow+\infty}\left|b_{k}\right|^{1 / k}=0\right\} .
\end{aligned}
$$

Именно классы $E_{\varphi, q}$ при соответствуюших ограничениях на функцию $\varphi$ и будут поставлять нам решения проблемы моментов (1).

Теорема. Пусть $q>1,\left\{\alpha_{n}\right\}_{n=1}^{\infty}-$ последовательность комплексных чисел, удовлетворяющая следуюшим условиям:

$$
\lim _{n \rightarrow \infty} \operatorname{Re} \alpha_{n}=+\infty
$$

и при всех $m \neq n$, дл которых $\operatorname{Re} \alpha_{n}=\operatorname{Re} \alpha_{m}$, имеем

$$
\frac{\left(\operatorname{Im} \alpha_{n}-\operatorname{Im} \alpha_{m}\right) \ln q}{2 \pi} \notin \mathbb{Z} .
$$

Пусть также $\varphi \in S_{0}$ и функиия $t^{\alpha} \varphi(t)$, где $\alpha=\min \operatorname{Re} \alpha_{n}$, ограничена на $(0,1)$.

Тогда для любой $\left\{c_{n}\right\}_{n=1}^{\infty} \in \mathbb{C}^{\infty}$ существует функиия $f \in E_{\varphi, q}$, удовлетворяюшая условиям

$$
\int_{0}^{+\infty} t^{\alpha_{n}} f(t) d t=c_{n} \quad \forall n \in \mathbb{N}
$$

в том и только том случае, когда при всех $n \in \mathbb{N}$

$$
\varphi_{n}=\int_{0}^{+\infty} t^{\alpha_{n}} \varphi(t) d t \neq 0
$$

ДокАЗАТЕльСтво. Если $q>1$ и последовательность $\left\{\alpha_{n}\right\}_{n=1}^{\infty}$ Удовлетворяют условиям (5) и (6), то все числа $\left\{q^{\alpha_{n}}\right\}_{n=1}^{\infty}$ различны и

$$
\lim _{n \rightarrow \infty} q^{\alpha_{n}}=\infty
$$

Следовательно, если $\varphi_{n}$ - моменты функции $\varphi \in S_{0}$ (см. (8)) - отличны от нуля, то согласно $\left[3\right.$, с. 297] для любой $\left\{c_{n}\right\}_{n=1}^{\infty} \in \mathbb{C}^{\infty}$ существует целая функция $g(z)=\sum_{k=0}^{\infty} a_{k} z^{k}$ такая, что

$$
g(0)=1, \quad g\left(q^{\alpha_{n}}\right)=\frac{c_{n}}{\varphi_{n}} \quad \forall n \in \mathbb{N} .
$$


Положим

$$
f(t)=\sum_{k=0}^{\infty} a_{k} q^{-k} \varphi\left(t q^{-k}\right) .
$$

Ввиду (10) и (4) $f \in E_{\varphi, q}$ Ясно, что при любом $\beta \in \mathbb{C}, b=\operatorname{Re} \beta \geqslant \alpha=$ $\min \operatorname{Re} \alpha_{n}$ последовательность функций

$$
t^{\beta} \sum_{k=0}^{N} a_{k} q^{-k} \varphi\left(t q^{-k}\right)
$$

сходится в каждой точке $t \in(0,+\infty)$ к $t^{\beta} f(t) \in C(0,+\infty)$ и ограничена по абсолютной величине функцией

$$
t^{b} \psi(t)=t^{b} \sum_{k=0}^{\infty}\left|a_{k} q^{-k} \varphi\left(t q^{-k}\right)\right| .
$$

Из условия теоремы и определения класса $S_{0}$ видно, что при всех $b \geqslant \alpha$ имеет место оценка

$$
\sup _{x>0}\left|x^{b} \varphi(x)\right|=M(b)<+\infty .
$$

А так как $\left\{a_{n}\right\}-$ коэффициенты Тейлора целой функции, то при любом $\gamma \in \mathbb{R}$

$$
\sum_{k=0}^{\infty}\left|a_{k} q^{k \gamma}\right|=C(\gamma)<+\infty .
$$

Отсюда при всех $b \geqslant \alpha$ находим

$$
\begin{aligned}
t^{b} \psi(t) & =\sum_{k=0}^{\infty}\left|a_{k} q^{-k} q^{k b}\right|\left(t q^{-k}\right)^{b}\left|\varphi\left(t q^{-k}\right)\right| \\
& \leqslant \sup _{x>0}\left|x^{b} \varphi(x)\right| \sum_{k=0}^{\infty}\left|a_{k} q^{k(b-1)}\right|=M(b) C(b-1) .
\end{aligned}
$$

Следовательно, функции $t^{b} \psi(t)$ ограничены на $(0,+\infty)$ при всех $b \geqslant \alpha$. А это влечет за собой включения

$$
t^{b} \psi(t) \in L_{1}(0,+\infty) \quad \forall b \geqslant \alpha .
$$

Ввиду (12) к последовательности функций (11) можно применить теорему Лебега о предельном переходе под знаком интеграла. Имеем

$$
\begin{aligned}
\int_{0}^{+\infty} t^{\beta} f(t) d t & =\sum_{k=0}^{\infty} a_{k} q^{-k} \int_{0}^{+\infty} t^{\beta} \varphi\left(t q^{-k}\right) d t \\
& =\sum_{k=0}^{\infty} a_{k} q^{k \beta} \int_{0}^{+\infty}\left(t q^{-k}\right)^{\beta} \varphi\left(t q^{-k}\right) d\left(t q^{-k}\right) \\
& =\sum_{k=0}^{\infty} a_{k} q^{k \beta} \int_{0}^{+\infty} x^{\beta} \varphi(x) d x=g\left(q^{\beta}\right) \int_{0}^{+\infty} t^{\beta} \varphi(t) d t
\end{aligned}
$$


Из (13) и (9) получаем (7). Если же для какого-то $n \in \mathbb{N}$ имеем равенство $\int_{0}^{+\infty} t^{\alpha_{n}} \varphi(t) d t=0$, то и для любой функции $f \in E_{\varphi, q}$ справедливо $\int_{0}^{+\infty} t^{\alpha_{n}} f(t) d t=0$. Следовательно, в этом случае, бесконечная система уравнений $(7)$ не может быть разрешима в классе $E_{\varphi, q}$ при любых правых частях. Теорема доказана.

Нетрудно сообразить, что для любой строго возрастающей последовательности вешественных чисел $\left\{\alpha_{n}\right\}$ выполнено (6), а соотношения (8) в этом случае будут справедливы для любой неотрицательной функции $\varphi \in S_{0}$, не равной нулю тождественно.

Сказанное позволяет без труда получить из теоремы

СлЕДСТВИЕ 1. Пусть $\left\{\alpha_{n}\right\}_{n=1}^{\infty}-$ произвольная строго возрастающая последовательность действительных чисел, $\lim _{n \rightarrow \infty} \alpha_{n}=+\infty$. Пусть также $\varphi \in S_{0}$ - неотрицательная на $(0,+\infty)$ функиия, не равная нулю тождественно, $u$, кроме того, функиия $t^{\alpha_{1}} \varphi(t)$ ограничена на $(0,1)$.

Тогда, каковы бы ни были $q>1 u\left\{c_{n}\right\}_{n=1}^{\infty} \in \mathbb{C}^{\infty}$, существует функция $f \in E_{\varphi, q}$ такая, что

$$
\int_{0}^{+\infty} t^{\alpha_{n}} f(t) d t=c_{n} \quad \forall n \in \mathbb{N} .
$$

Следствие 1 , в свою очередь, позволяет получить ряд предложений, усиливаюших результаты работ [1] и [2].

СлеДСТВИЕ 2. Для любой $\left\{c_{n}\right\}_{n=0}^{\infty} \in \mathbb{C}^{\infty}$ существует иелая функиия $f$ порядка 2 типа 1 , удовлетворяющая условиям (2).

СледСтвиЕ 3. Пусть $1 / 2<\rho<+\infty,\left\{\alpha_{n}\right\}_{n=1}^{\infty}-$ произвольная строго возрастающая последовательность действительных чисел, $\lim _{n \rightarrow \infty} \alpha_{n}=+\infty$. Тогда для любой $\left\{c_{n}\right\}_{n=1}^{\infty} \in \mathbb{C}^{\infty}$ и любого $\sigma$, $0<\sigma<+\infty$, существует целая функиия $f$ порядка $\rho$ типа $\sigma$, для которой выполняются соотношения (14).

Через $\mathscr{A}(\mathscr{D})$ обозначим множество всех функций, аналитичных в области $\mathscr{D}$.

СлЕДСТВИЕ 4. Какова бы ни была строго возрастающая последовательность действительных чисел $\alpha_{n}, \lim _{n \rightarrow \infty} \alpha_{n}=+\infty$, для любой $\left\{c_{n}\right\}_{n=1}^{\infty} \in \mathbb{C}^{\infty}$ существует функиия $f \in S_{+} \cap \mathscr{A}(\mathbb{C} \backslash(-\infty, 0])$, удовлетворяющая условиям (14).

Если в следствии 4 положить $\alpha_{n}=n-1, n \in \mathbb{N}$, то получим предложение, усиливающее приведенньй вьше результат из [2].

СлеДСТВИЕ 5. Для любой $\left\{c_{n}\right\}_{n=0}^{\infty} \in \mathbb{C}^{\infty}$ существует функиия $f \in S_{+} \cap \mathscr{A}(\mathbb{C} \backslash(-\infty, 0])$, для которой выполняются равенства (3).

Теперь из следствия 1 выведем следствия $2-4$. Поясним основную идею их доказательств. Приводимые ниже леммы 1-3 устанавливают, что функции из множеств $E_{\varphi, q}$ наследуют ряд аналитических свойств функции $\varphi$. 
Поэтому согласно следствию 1 для доказательства разрешимости проблемы моментов

$$
\int_{0}^{+\infty} t^{\alpha_{n}} f(t) d t=c_{n}, \quad n=1,2, \ldots,
$$

где $\alpha_{n} \uparrow+\infty$, при любых правых частях $\left\{c_{n}\right\}$ в том или ином классе функций $X$, оказывается достаточным установить справедливость следующих двух утверждений:

1) хотя бы при одном значении $q>1$

$$
\forall \varphi \in X \Longrightarrow E_{\varphi, q} \subset X
$$

$2)$ в классе $X$ содержится функция $\varphi \in S_{0}$, неотрицательная на $(0,+\infty)$ и не равная нулю тождественно.

Через $[\rho, \sigma), 0<\rho<+\infty, 0<\sigma<+\infty$, обозначим множество целых функций, порядок которых или меньше $\rho$, или равен $\rho$, но тип меньше $\sigma$.

Лемма 1. Пусть $q>1,0<\rho<+\infty, 0<\sigma<+\infty, \varphi-$ иелая функиия порядка $\rho$ типа $\sigma, f \in E_{\varphi, q}$. Тогда

$$
u(t)=f(t)-\varphi(t) \in[\rho, \sigma) .
$$

ДоКАЗАТЕЛЬСТво. Из (4) следует, что функция $u(t)$ имеет вид

$$
u(t)=\sum_{k=1}^{\infty} b_{k} \varphi\left(t q^{-k}\right) .
$$

Поскольку

$$
\max _{|t| \leqslant R} \max _{k \in \mathbb{N}}\left|\varphi\left(t q^{-k}\right)\right|=\max _{|z| \leqslant R q^{-1}}|\varphi(z)|,
$$

a $\sum_{k=1}^{\infty}\left|b_{k}\right|=b<+\infty$, то заключаем, что ряд (15) сходится равномерно в любом круге $|t| \leqslant R$, и, кроме того, при любом $R>0$ справедливо неравенство

$$
\max _{|z| \leqslant R}|u(z)| \leqslant b \max _{|z| \leqslant R q^{-1}}|\varphi(z)| .
$$

Отсюда вытекает утверждение леммы.

ДокАЗАТЕльСТво следствия 2. Положим

$$
\varphi_{j}(t)=t^{j} \exp \left(-t^{2}\right), \quad j=0,1, \quad \alpha_{n}=n-1, \quad n \in \mathbb{N} .
$$

Возьмем и зафиксируем некоторое $q>1$. Нетрудно видеть, что все условия следствия 1 для последовательности $\left\{\alpha_{n}\right\}$ и функций $\varphi_{j}, j=0,1$, выполняются. Следовательно, для любой $\left\{c_{n}\right\}_{n=0}^{\infty} \in \mathbb{C}^{\infty}$ существуют функции $f_{j} \in E_{\varphi_{j}, q}, j=0,1$, для которых справедливы равенства

$$
\int_{0}^{+\infty} t^{n} f_{j}(t) d t=\frac{c_{n}}{2} \quad \forall n \in \mathbb{N}_{0}, \quad j=0,1 .
$$


Теперь положим $f(t)=f_{0}(t)+f_{1}(t)$. По лемме 1

$$
f(t)=\varphi_{0}(t)+u_{0}(t)+\varphi_{1}(t)+u_{1}(t),
$$

где $u_{0}(t), u_{1}(t) \in[2,1)$. Следовательно,

$$
f(t)=(1+t) \exp \left(-t^{2}\right)+u(t),
$$

где $u(t)=u_{0}(t)+u_{1}(t) \in[2,1)$. Отсюда заключаем, что $f$ является целой функцией порядка 2 типа 1 . Так как функция $f_{0}$ четна, а $f_{1}$ нечетна, то из (16) находим

$$
\int_{-\infty}^{0} t^{n} f_{j}(t) d t=(-1)^{n+j} \frac{c_{n}}{2} \quad \forall n \in \mathbb{N}_{0}, \quad j=0,1 .
$$

Складывая соотношения (16) и (17), приходим к равенствам

$$
\int_{-\infty}^{+\infty} t^{n} f(t) d t=\frac{c_{n}}{2}\left(2+(-1)^{n}+(-1)^{n+1}\right)=c_{n} \quad \forall n \in \mathbb{N}_{0} .
$$

Следствие 2 доказано.

ДокАЗАТЕЛЬСТво следствия 3 . При всех $\rho, 1 / 2<\rho<+\infty$, существует целая функция $F_{\rho}$ порядка $\rho$, имеюшая конечньй и положительньй тип, неотрицательная на $(0,+\infty)$ и лежащая в $S_{0}$. Действительно, если $\rho=1$, то указанным условиям удовлетворяет функция $\exp (-z)$, а если $\rho>1 / 2$ и $\rho \neq 1$, то полагаем

$$
F_{\rho}(z)=\prod_{n=1}^{\infty}\left(1-z^{m} n^{-\tau}\right)^{2},
$$

где $m=[\rho]+1$, а $\tau=m / \rho$. Из результатов, имеющихся в [4, с. 290-304], следует, что функция (18) обладает всеми требуемьми свойствами.

Возьмем $N \in \mathbb{N}$ таким, чтобы $N+\alpha_{1}>0$. Из сказанного вытекает, что если заданы числа $\rho>1 / 2$ и $\sigma>0$, то при некотором $\gamma>0$ функция

$$
\varphi(t)=t^{N} F_{\rho}(\gamma t)
$$

имеет порядок $\rho$ и тип $\sigma$, неотрицательна на $(0,+\infty)$ и лежит в $S_{0}$. При этом, очевидно, функция $t^{\alpha_{1}} \varphi(t)$ ограничена на $(0,1)$. Зафиксируем некоторое $q>1$. Тогда согласно следствию 1 для любой $\left\{c_{n}\right\}_{n=1}^{\infty} \in \mathbb{C}^{\infty}$ существует $f \in E_{\varphi, q}$, удовлетворяюшая условиям (14). По лемме $1 f(t)=$ $\varphi(t)+u(t)$, причем $u \in[\rho, \sigma)$. Следовательно, $f$ - целая функция порядка $\rho$ типа $\sigma$. Следствие 3 доказано.

ЛЕмма 2. Если $q>1, \varphi \in S_{+}$, mo $E_{\varphi, q} \subset S_{+}$. 
ДокАЗАТЕЛЬСТво. Поскольку $\varphi \in S_{+}$, то $\forall m \in \mathbb{N}_{0} \varphi^{(m)} \in S_{0}$. Поэтому $m$ раз продифференцированные ряды вида (4) сходятся равномерно на $[0,+\infty)$ и являются рядами такого же вида. Тем самым, для любой функции $f \in E_{\varphi, q}$ справедливы включения

$$
f \in \mathbb{C}^{\infty}[0,+\infty) \quad \forall m \in \mathbb{N}_{0}, \quad f^{(m)} \in E_{\varphi, q} .
$$

В доказательстве теоремы мы фактически проверили, что для любых $\psi \in S_{0}$ и $q>1$ множество $E_{\psi, q}$ содержится в $S_{0}$. Это вместе с (19) дает

$$
f^{(m)} \in S_{0} \quad \forall m \in \mathbb{N}_{0} .
$$

Подставив $t=0$ в продифференцированные ряды для функции $f$, убеждаемся в том, что соотношения

$$
\varphi^{(m)}(0)=0 \quad \forall m \in \mathbb{N}_{0}
$$

влекут за собой равенства

$$
f^{(m)}(0)=0 \quad \forall m \in \mathbb{N}_{0} .
$$

Из (20) и (21) получаем утверждение леммы.

Через $H_{\infty}\left(\Delta_{\theta}\right), 0<\theta<\pi$, обозначим множество всех функций, аналитических и ограниченных в угле

$$
\Delta_{\theta}=\{z \in \mathbb{C} \mid-\theta<\arg z<\theta, z \neq 0\} .
$$

ЛЕмма 3. Пусть $q>1, \varphi \in \bigcap_{0<\theta<\pi} H_{\infty}\left(\Delta_{\theta}\right)$. Тогда

$$
E_{\varphi, q} \subset \mathscr{A}(\mathbb{C} \backslash(-\infty, 0]) \text {. }
$$

ДОКАЗАТЕЛЬСТВО. ПоЛОЖим

$$
M_{\theta}=\sup _{z \in \Delta_{\theta}}|\varphi(z)|<+\infty, \quad \theta \in(0, \pi) .
$$

Заметим, что если $\theta \in(0, \pi), z \in \Delta_{\theta}$, то при всех $k \in \mathbb{N}$ имеем $z q^{-k} \in \Delta_{\theta}$. Поэтому

$$
\sup _{k \in \mathbb{N}} \sup _{z \in \Delta_{\theta}}\left|\varphi\left(z q^{-k}\right)\right|=M_{\theta}
$$

Ввиду сказанного все ряды вида

$$
\sum_{k=0}^{\infty} b_{k} \varphi\left(z q^{-k}\right), \quad \text { где } \sum_{k=0}^{\infty}\left|b_{k}\right|<+\infty,
$$

равномерно сходятся в углах $\Delta_{\theta} \forall \theta \in(0, \pi)$. Следовательно, для любой функции $f \in E_{\varphi, q}$ справедливы включения

$$
f \in \bigcap_{0<\theta<\pi} H_{\infty}\left(\Delta_{\theta}\right) \subset \mathscr{A}(\mathbb{C} \backslash(-\infty, 0]) .
$$


Лемма доказана.

Если $\varphi \in S_{+}$, то $t^{a} \varphi(t) \in S_{0}$ при любом $a \in \mathbb{R}$. Поэтому для того, чтобы вывести следствие 4 из следствия 1 и лемм 2 и 3 , достаточно доказать существование положительной на $(0,+\infty)$ функции $\varphi$, для которой одновременно вьполняются включения

$$
\varphi \in \bigcap_{0<\theta<\pi}^{\varphi \in S_{+},} H_{\infty}\left(\Delta_{\theta}\right) .
$$

В качестве такой функции $\varphi$ можно взять

$$
\varphi(t)= \begin{cases}1 / B(t), & t \in \mathbb{C} \backslash(-\infty, 0] \\ 0, & t=0\end{cases}
$$

где $B(t)=\sum_{n=-\infty}^{+\infty} 2^{-n(n-1)} t^{n}$. Включение (23) следует из разложения функции $B(t)$ в бесконечное произведение, найденного в [5, лемма 3].

Для доказательства (22) достаточно проверить, что для функции $\varphi$, определенной в $(24)$, вьполняются равенства

$$
\begin{gathered}
\varphi^{(k)}(0)=0 \quad \forall k \in \mathbb{N}_{0}, \\
\lim _{t \rightarrow+\infty} t^{m} \varphi^{(k)}(t)=0 \quad \forall m, k \in \mathbb{N}_{0} .
\end{gathered}
$$

Установим справедливость (26). С этой целью будет доказана

Лемма 4. При любых $n \in \mathbb{N} u x>x_{0}(n)$ выполняется неравенство

$$
0<B^{(n)}(x)<2 B(x) .
$$

ДокАЗАТЕЛЬСТво. Имеем $B(x)=B^{+}(x)+B^{-}(x)$, где

$$
B^{+}(x)=\sum_{m=0}^{\infty} 2^{-m(m-1)} x^{m}, \quad B^{-}(x)=\sum_{m=-\infty}^{-1} 2^{-m(m-1)} x^{m} .
$$

$\Phi$ ункция $B^{-}(z)$ является аналитической в области $|z|>0$ и равна нулю на бесконечности. Следовательно, и все ее производные обладают этим же свойством. В то же время функция $B^{+}(x)$ при $x \in \mathbb{R}, x \rightarrow+\infty$, неограниченно возрастает со всеми своими производными. Ввиду сказанного, для доказательства соотношения (27) достаточно при $x \geqslant 0$ установить неравенства

$$
B^{+^{(n)}}(x) \leqslant B^{+}(x) \quad \forall n \in \mathbb{N} .
$$

Легко проверить, что если последовательность $\left\{a_{n}\right\}_{n=0}^{\infty}$ положительна и не возрастает, то функция $F(z)=\sum_{n=0}^{\infty} a_{n} z^{n} / n$ ! является целой, все ее производные на луче $(0,+\infty)$ положительны и

$$
\forall x \geqslant 0, \quad \forall n \in \mathbb{N} \quad F^{(n)}(x) \leqslant F(x) .
$$


Это замечание вместе с очевидным соотношением $n ! \cdot 2^{-n(n-1)} \searrow 0$ приводит нас к (28). Тем самым лемма 4 доказана.

Имеем

$$
\varphi^{(n)}(t)=\frac{P_{n}\left(B(t), B^{\prime}(t), \ldots, B^{(n)}(t)\right)}{(B(t))^{n+1}},
$$

где $P_{n}$ - некоторьй однородный многочлен от $n+1$ переменной степени $n$. Поэтому, учитьвая (27), получаем оценку

$$
\left|P_{n}\left(B(t), B^{\prime}(t), \ldots, B^{(n)}(t)\right)\right|=O\left((B(t))^{n}\right), \quad t \rightarrow+\infty .
$$

Из (29), (30) и очевидного включения

$$
\varphi(t)=\frac{1}{B(t)} \in S_{0}
$$

выводим (26). Из (26) и легко проверяемого тождества $\varphi(1 / z)=z \varphi(z)$ следует (25). Требуемые свойства функции $\varphi(t)$ установлены, а это доказывает следствие 4 .

Московский государственный университет Поступило им. М.В. Ломоносова

Исправленный вариант

02.10 .95

\section{СПИСОК ЦИТИРОВАННОЙ ЛИТЕРАТУРЫ}

[1] Polya G. Sur l'indetermination d'un problème voisin du problème des moments // C. R. Acad. Sci. Paris. 1938. V. 207. P. 708-711.

[2] Duran A. J. The Stieltjes moments problem for rapidly decreasing functions // Proc. Amer. Math. Soc. 1989. V. 107. P. 731-741.

[3] Маркушевич А. И. Теория аналитических функций. Т. 2. М.: Наука, 1968.

[4] Титчмарш Е. Теория функций. М.-Л.: ГИТТЛ, 1951.

[5] Казьмин Ю.А. Об одной задаче А.О. Гельфонда // Матем. сб. 1973. T. 90 (132). № 4. C. 521-543. 